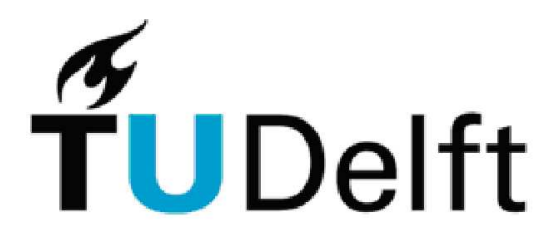

TU Delft Library

Prometheusplein 1

Postbus 98

2600 MG Delft

Tel: +31 (0) 152785678

Fax: +31 (0) 152785706

Email: library@tudelft.nl

www.library.tudelft.nl

Aan: T.N.O.

CREDITEURENADMINISTRATIE

POSTBUS 6061

2600 JA DELFT

NEDERLAND

Aanvraag nr: 1638423

Uw referentie(s): TS41021031 - H

Artikelomschrijving:

Aantal kopieën: 6

Artikel:

AN ORGANIC FERROELECTRIC FIELD EFFECT TRANSISTOR W

Auteur:

CAI

Titel:

APPLIED PHYSICS LETTERS

Jaar:

2014

Vol. 105

Nr. 11

Pag. 113113

Plaatsnummer: 784 B 


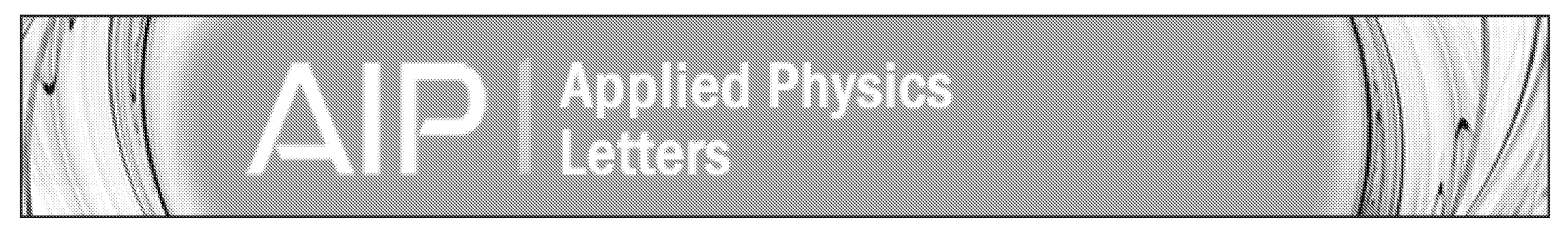

\section{An organic ferroelectric field effect transistor with poly(vinylidene fluoride-co- trifluoroethylene) nanostripes as gate dielectric}

Ronggang Cai, Hailu G. Kassa, Alessio Marrani, Albert J. J. M. van Breemen, Gerwin H. Gelinck, Bernard Nysten, Zhijun Hu, and Alain M. Jonas

Citation: Applied Physics Letters 105, 113113 (2014); doi: 10.1063/1.4896292

View online: http://dx.doi.org/10.1063/1.4896292

View Table of Contents: http://scitation.aip.org/content/aip/journal/apl/105/11?ver=pdfcov

Published by the AIP Publishing

\section{Articles you may be interested in}

Low-voltage-operated top-gate polymer thin-film transistors with high capacitance poly(vinylidene fluoridetrifluoroethylene)/poly(methyl methacrylate) dielectrics

J. Appl. Phys. 108, 102810 (2010); 10.1063/1.3511697

Erratum: "Poly(3-hexylthiophene) crystalline nanoribbon network for organic field effect transistors" [Appl. Phys. Lett.96, 243304 (2010)]

Appl. Phys. Lett. 97, 189902 (2010); 10.1063/1.3512957

Displacement current analysis of carrier behavior in pentacene field effect transistor with poly (vinylidene fluoride and tetrafluoroethylene) gate insulator

J. Appl. Phys. 106, 024505 (2009); 10.1063/1.3176487

Interface screening and imprint in poly(vinylidene fluoride/trifluoroethylene) ferroelectric field effect transistors J. Appl. Phys. 105, 054110 (2009); 10.1063/1.3088887

Electric field induced structural change for poly(vinylidene fluoride-co-trifluoroethylene) ultrathin films studied by scanning Maxwell stress microscope

J. Vac. Sci. Technol. B 16, $121(1998) ; 10.1116 / 1.589765$

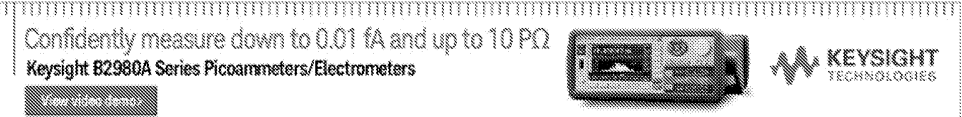




\title{
An organic ferroelectric field effect transistor with poly(vinylidene fluoride-co-trifluoroethylene) nanostripes as gate dielectric
}

\author{
Ronggang Cai, ${ }^{1, a)}$ Hailu G. Kassa, ${ }^{1}$ Alessio Marrani, ${ }^{2}$ Albert J. J. M. van Breemen, ${ }^{3}$ \\ Gerwin H. Gelinck, ${ }^{3}$ Bernard Nysten, ${ }^{1}$ Zhijun Hu, ${ }^{4}$ and Alain M. Jonas ${ }^{1, a)}$ \\ ${ }^{1}$ Bio \& Soft Matter, Institute of Condensed Matter and Nanosciences, Université catholique de Lowvain, \\ Croix du Sud 1/L7.04.02, B1348 Louvain-la-Neuve, Belgium \\ ${ }^{2}$ Solvay Specialty Polymers Italy S.P.A., Research and Development Center, Viale Lombardia, No. 20 , \\ Bollate (MI) 20021, Italy \\ ${ }^{3}$ Holst Centre/TNO; High Tech Campus 31, Eindhoven, The Netherlands \\ ${ }^{4}$ Center for Soft Condensed Matter Physics and Interdisciplinary Research, Soochow University, \\ Suzhou 215006, China
}

(Received 11 July 2014; accepted 10 September 2014; published online 19 September 2014)

\begin{abstract}
We demonstrate the fabrication of an organic Ferroelectric Field Effect Transistor (FeFET) incorporating a ferroelectric gate dielectric made of nanostripes obtained by nanoimprinting poly(vinylidene fluoride-co-trifluoroethylene) over a layer of semiconducting poly(triarylamine). The imprinting process results in a decreased switching voltage for the ferroelectric, by a factor of ca. 1.5, resulting in a decreased operating voltage compared to a reference FeFET with a continuous ferroelectric layer. The transistor consists of a large number of nanostripe-gated transistors placed in parallel, which also offers interesting possibilities for a strong size reduction of organic FeFETs. (C) 2014 AIP Publishing LLC. [http://dx.doi.org/10.1063/1.4896292]
\end{abstract}

Organic Ferroelectric Field Effect Transistors (FeFETs) incorporating a poly(vinylidene fluoride-co-trifluoroethylene) (P(VDF-TrFE)) as gate dielectric, have been intensively studied for non-volatile memory applications over the last decade. ${ }^{1-15} \mathrm{P}$ (VDF-TrFE) crystallizes predominantly in a polar ferroelectric phase characterized by a remnant polarization of ca. $70 \mathrm{mC} / \mathrm{m}^{2}$ (for $\sim 30 \% \mathrm{TrFE}$ ) and a coercive field of $\mathrm{ca}$. $50 \mathrm{MV} / \mathrm{m}$. By applying a gate voltage $V_{g s}$, the P(VDF-TrFE) is permanently polarized, resulting in the accumulation of charge carriers in the semiconducting layer at its interface with the P(VDF-TrFE) layer even after gate voltage removal, i.e., at $V_{g s}=0 \mathrm{~V}$. The source-drain current $I_{d s}$, which is measured by applying a small source-drain voltage $V_{d s}$ together with a small gate voltage $V_{g s}$, therefore, exhibits two current states, $I_{o n}$ and $I_{o f f}$, depending on the direction of polarization of P(VDF-TrFE) and on the semiconductor type. Since FeFET memories typically require smaller $V_{g s}$ and higher ON/ OFF current ratios, several methodologies have been explored to improve performance, including using high mobility semiconductors, improving the ferroelectric properties of P(VDF-TrFE), or optimizing the quality of the semiconductor/ P(VDF-TrFE) interface. $5,8,9,11,16,17$

Here, we focus on exploring the effect of nanoimprinting lithography (NIL) on the ferroelectric performance of P(VDF-TrFE) in an organic FeFET. We have previously reported that, when $\mathrm{P}(\mathrm{VDF}-\mathrm{TrFE})$ crystallizes in confinement in the nanocavities or nanochannels of a NIL mold, preferred crystallographic orientation and a significant increase of crystal perfection are observed; as a result, the coercive field of the material is significantly decreased. ${ }^{18,19}$ Similar improvements were also reported for the crystallization of $\mathrm{P}(\mathrm{VDF}-\mathrm{TrFE})$ in other confined media. ${ }^{20-22}$ Here, we

\footnotetext{
${ }^{a}$ Authors to whom correspondence should be addressed. Electronic addresses: ronggang.cai@uclouvain.be and alain.jonas@uclouvain.be
}

integrate nanoimprinting of P(VDF-TrFE) in the FeFET fabrication process, in order to decrease the gate voltage $V_{g s}$ needed to reach the saturation remnant polarization $P_{r} ;$ this concept simultaneously leads to an unusual FeFET wherein the gate ferroelectric is in the form of nanostripes running perpendicular to source and drain (Figure 1).

P(VDF-TrFE) with $35 \mathrm{wt}$. \% of TrFE units and a weightaverage molar mass of $c a .300000 \mathrm{~g} / \mathrm{mol}$ (Figure 1) was provided by Solvay Specialty Polymers. Poly(triarylamine) (PTAA, Figure 1) was purchased from Flexink. P(VDFTrFE) was dissolved in acetylacetone at different concentrations $(35 \mathrm{~g} / \mathrm{l}$ or $60 \mathrm{~g} / \mathrm{l})$. PTAA was dissolved in toluene $(10 \mathrm{~g} / \mathrm{l})$. All solutions were filtered by PTFE filters with pore size of $0.45 \mu \mathrm{m}$ before spincoating.

Source and drain electrodes were defined by standard photolithography on a Si (100) substrate covered by $200 \mathrm{~nm}$ of thermally grown $\mathrm{SiO}_{2}$, followed by the local etching of $30 \mathrm{~nm} \mathrm{SiO} 2$, the deposition of $5 / 25 \mathrm{~nm} \mathrm{Cr} / \mathrm{Au}$, and metal liftoff. These processes led to buried interdigitated source-drain electrodes in an almost perfectly planar substrate. The PTAA solution was spincoated at $2000 \mathrm{rpm}$ for $1 \mathrm{~min}$ on this substrate, resulting in the deposition of $c a .45 \mathrm{~nm}$ PTAA. A $35 \mathrm{~g} / \mathrm{l}$ $\mathrm{P}$ (VDF-TrFE) solution was then spincoated at $3500 \mathrm{rpm}$ for 1 min on the PTAA layer. Acetylacetone, used to dissolve P(VDF-TrFE), is an orthogonal solvent and hence does not affect the underlying PTAA layer. The P(VDF-TrFE) film, of ca. $95 \mathrm{~nm}$ initial thickness, was nanoimprinted under 60 bars at $135^{\circ} \mathrm{C}$ (in-between the Curie transition and the melting point) with an Obducat imprinter. ${ }^{23}$ After 10 min imprinting time, the system was cooled to room temperature. During imprinting, the line direction was placed normal to the sourcedrain direction. After imprinting, the resulting P(VDF-TrFE) nanostripes were of $c a .200 \mathrm{~nm}$ width, $200 \mathrm{~nm}$ spacing, and $185 \mathrm{~nm}$ height. They were effectively isolated from each other, since the initial thickness of the P(VDF-TrFE) layer 
<smiles>Cc1ccc(N(c2ccc(C)cc2C)c2c(C)cc(C)cc2C)cc1</smiles><smiles>CC(C)(C)C(F)(F)C(C)(F)C(C)(F)C(F)(F)F</smiles>

FIG. 1. Structures of PTAA and P(VDF-TrFE), and scheme of a FeFET with nanoimprinted ferroelectric. In case of the reference FeFET, the imprinted P(VDF-TrFE) was replaced by a continuous film. was selected to be $c a .95 \mathrm{~nm}$ in order to ensure nanoimprinting in complete confinement. For the reference device, a $60 \mathrm{~g} / \mathrm{l}$ $\mathrm{P}$ (VDF-TrFE) solution was spincoated at $3500 \mathrm{rpm}$ for $1 \mathrm{~min}$ on the PTAA layer, resulting in a film of $c a .185 \mathrm{~nm}$ thickness, equal to the height of the ferroelectric nanostripes in the imprinted samples. This reference device was annealed at $135^{\circ} \mathrm{C}$ for $10 \mathrm{~min}$ on a hot plate to improve crystallinity. The thicknesses of the PTAA layer and of the P(VDF-TrFE) layers were measured by Atomic Force Microscopy (AFM).

The morphology of the continuous films and nanoimprinted nanostripes was checked by a Multimode AFM (Nanoscope IV, Bruker) in tapping mode with silicon cantilevers from Nanosensors (force constant of $\sim 40 \mathrm{~N} / \mathrm{m}$, apex radius of curvature $<7 \mathrm{~nm}$ ). The piezoresponse of the continuous and nanoimprinted P(VDF-TrFE) films was checked by Piezoresponse Force Microscopy (PFM) in contact mode. An Agilent 5500 AFM (Agilent Technologies) system equipped with a Mac III Controller lock-in amplifier was adapted to the requirements of the PFM technology, in order to apply dc biases larger than $10 \mathrm{~V}$. To make PFM measurements, the source-drain electrodes were used as bottom electrodes. A conductive Si cantilever from Nanosensors (Boron-doped diamond-coated, force constant of $0.02-0.77 \mathrm{~N} / \mathrm{m}$ ) was used as a mobile top electrode. The ferroelectric response was inspected in PFM image mode with an oscillating voltage of $0.5 \mathrm{~V}$ amplitude, added to a polarizing dc bias. All PFM measurements were done by applying the ac driving bias $V_{\text {tip }}$ on the cantilever, while the poling was done by applying the dc bias $V_{s \& d}$ on the source-drain electrodes and setting the ac bias to zero (Figure 2(a)). Here we define the poling voltage $V_{\text {tip }, p o l}=V_{g s}=V_{\text {tip }}-V_{s \& d}=-V_{s \& d}$.

P(VDF-TrFE) was first poled by applying a poling voltage $V_{\text {tip,pol }}$. Then the source-drain current $I_{d s}$ was measured after the removal of $V_{\text {tip,pol }}$. As shown in Figure 2(a), the same setup configuration as for PFM measurements was used to pole P(VDF-TrFE). Then a Keithley analyzer (Model 6430 with femtoampere accuracy) was used to apply a source-drain voltage $V_{d s}$ and measure the source-drain current $I_{d s}$ (Figure 2(b)).
Figure 3 shows the topography of the surfaces along the different fabrication steps. To avoid contamination and damage to the NIL molds, the Au electrodes should not be higher than the $\mathrm{SiO}_{2}$ part. Therefore, the Au electrodes were buried into the substrate as mentioned above. Figures $3(a)$ and $3(e)$ show such a substrate with Au electrodes at nearly the same level as the $\mathrm{SiO}_{2}$ background. Deposition of the PTAA layer flattens further the topography (Figures $3(\mathrm{~b})$ and 3(f)). A surface devoid of any topology of electrodes is obtained after spincoating ca. $95 \mathrm{~nm}$ P(VDF-TrFE) on PTAA (Figures 3(c) and $3(\mathrm{~g}))$ : the average roughness is $8.0 \mathrm{~nm}$, comparable to the values reported for a $\mathrm{P}(\mathrm{VDF}-\mathrm{TrFE})$ film spin-coated on a flat surface. ${ }^{2,7}$ NIL applied on this P(VDF-TrFE) film produces well-defined nanostripes of $c a .185 \mathrm{~nm}$ height and $200 \mathrm{~nm}$ width (Figures $3(\mathrm{~d})$ and $3(\mathrm{~h})$ ). The nanostripes were imprinted perpendicularly to the source and drain electrodes. Due to tearing during demolding, and mold imperfections, a few defects were distributed along the array of nanostripes; Figure 3(d) shows a typical image. After optimization, the process led to well-structured nanoimprinted FeFETs with a reasonable production yield $(>90 \%)$. For current characterization, a conductive PFM cantilever was used as gate instead of a traditional metal electrode. The schematic diagram of such a nanoimprinted FeFET with top-gate bottom-contact configuration and mobile gate is shown in Figure 2(a).

The switching of the continuous and nanoimprinted P(VDF-TrFE) films atop PTAA in the FeFET architecture was studied by PFM: a series of regions of the sample were poled by scanning the grounded tip while applying increasing dc voltages $V_{s \& d}$ to the source and drain electrodes; the sample was then imaged in PFM mode at zero dc bias. Figures $4(a)$ and $4(b)$ show the resulting amplitude images, Figs. $4(\mathrm{c})$ and $4(\mathrm{~d})$ phase images, of the continuous P(VDF-TrFE) film (Figs. 4(a) and $4(\mathrm{c})$ ) and of the nanoimprinted P(VDF-TrFE) film (Figs. 4(b) and 4(d)). For both films, the piezoresponse changes depending on the previously applied $V_{s \& d} .10 \mathrm{~V}$ is insufficient to align the dipoles of P(VDF-TrFE); however, starting from $15 \mathrm{~V}$ for the continuous film and $12.5 \mathrm{~V}$ for the nanoimprinted film, the PFM contrast starts to appear
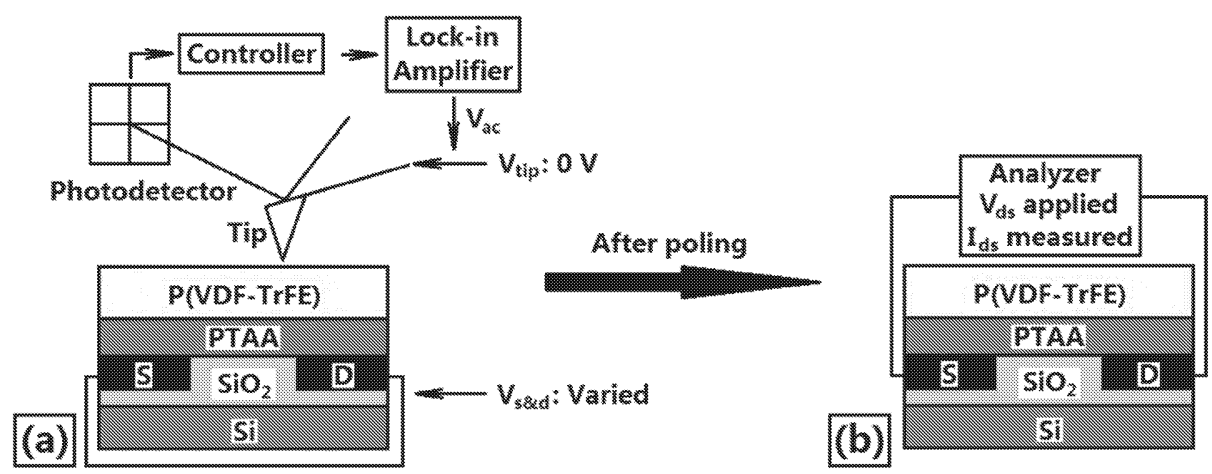

FIG. 2. Configuration of the setups for (a) applying $V_{\text {tip.pol }}$ to pole $\mathrm{P}$ (VDF-TrFE) and (b) applying $V_{d s}$ to measure $I_{d s}$. 

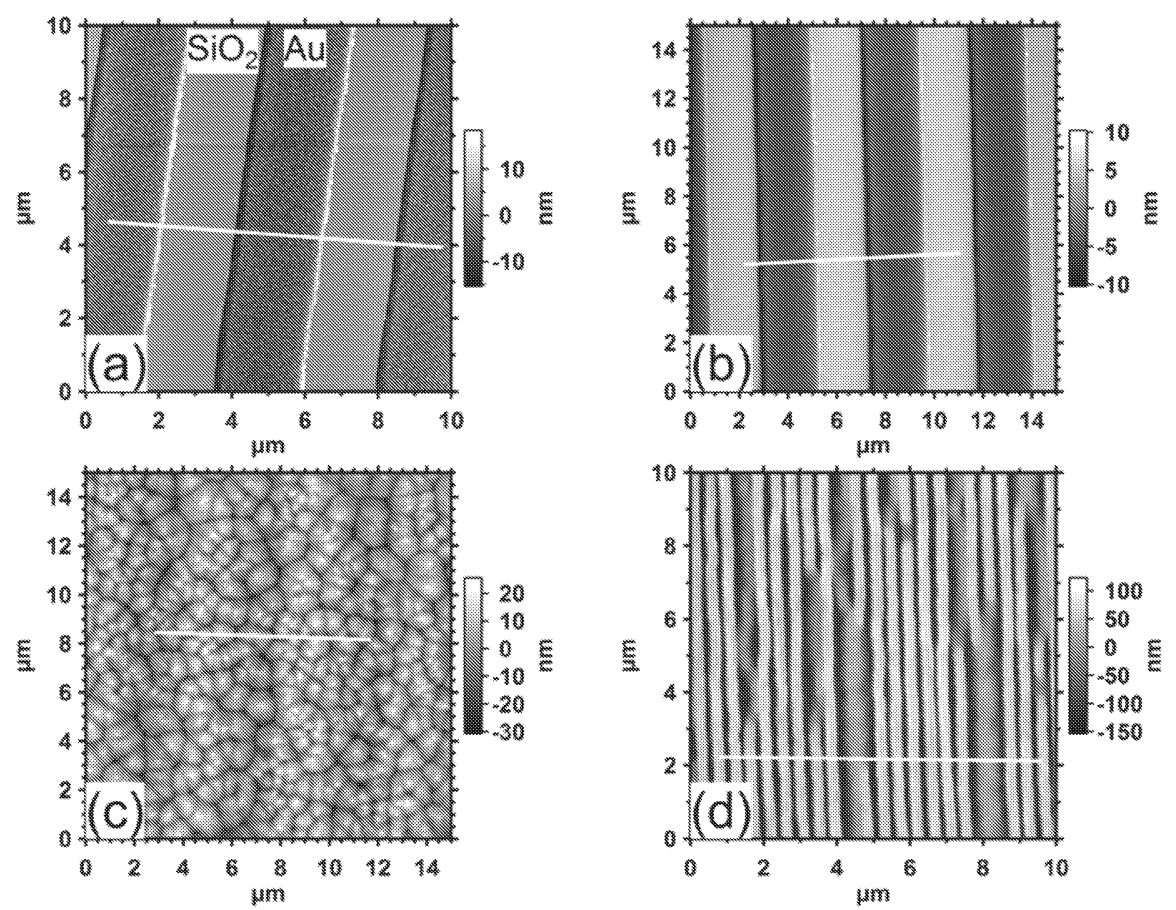

FIG. 3. AFM morphology of: (a) buried S/D substrate; (b) PTAA layer spincoated on (a); (c) P(VDF-TrFE) layer spincoated on (b); (d) P(VDFTrFE) nanostripes by making nanoimprint on (c); (e)-(h) height profiles of the cross sections of (a)-(d): (e) corresponds to (a), ( $f$ ) to (b), (g) to (c), and (h) to (d).
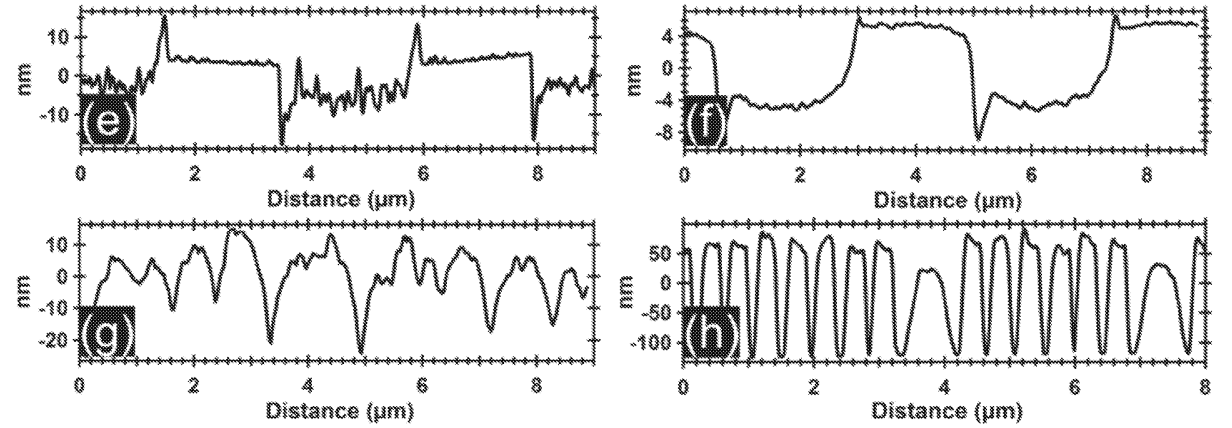

due to partial dipole alignment. The contrast increases for larger positive $V_{s \& d}$ and reaches a maximum when $V_{s \& d}$ is large enough to permanently align most dipoles in one direction. The maximum contrast means that the saturation $P_{r}$ is obtained.

To determine at which voltage the maximum $P_{r}$ can be obtained, the phase contrast was plotted versus $V_{s \& d}$ in Figures $4(e)$ and $4(f)$ for both the continuous and nanoimprinted P(VDF-TrFE) films. Here, the phase contrast was defined as $A_{0} \cos \varphi_{0}-A \cos \varphi$, where $A_{0}$ and $\varphi_{0}$ are the average amplitude and the average phase of the background; $A$ and $\varphi$ are the average amplitude and phase of the smaller square areas where $V_{s \& d}$ was applied. As shown in Figures $4(\mathrm{e})$ and $4(\mathrm{f})$, the contrast reaches a maximum at $27.5 \mathrm{~V}$ and a plateau is obtained for voltages larger than $27.5 \mathrm{~V}$ for the continuous $\mathrm{P}$ (VDF-TrFE) film, while this point is $20 \mathrm{~V}$ for the nanoimprinted P(VDF-TrFE) film. Note that the thickness of the continuous film was selected so as to be equivalent to the height of the nanostripes of the nanoimprinted sample. This means that the switching field of the nanoimprinted $\mathrm{P}(\mathrm{VDF}-\mathrm{TrFE})$ is indeed lower than the one of the continuous film. For instance, at $15 \mathrm{~V}, \mathrm{ca} .5 \%$ of the maximum polarization contrast is obtained for the continuous film, whereas ca. $60 \%$ is obtained for the nanoimprinted film. Hence, the P(VDF-TrFE) dipoles are more easily aligned in nanostripes than in the continuous film, with a switching voltage reduced by a factor of about 1.5 .
Previously, we showed that the coercive field of P(VDFTrFE) imprinted over Si in nanostripes of similar width was reduced by a factor of about 2 compared to a continuous film. ${ }^{19}$ The lower effect found here might be due to the different nature of the bottom electrode.

As before, a conductive cantilever was used as gate for the characterization of the device. The grounded tip was scanned over a defined area of $50 \mu \mathrm{m} \times 50 \mu \mathrm{m}$ while setting $V_{s \& d}$ to a given value, in order to pole the system at $V_{t i p, p o l}=-V_{s \& d}$. Then, the polarization-induced source-drain current $I_{p o l}$ was measured as displayed in Figure 2(b), for different source-drain voltages $V_{s d}$ and no applied gate voltage. Here, $I_{p o l}$ is defined as $I_{p o l}=I_{d s}-I_{\text {initial }}$, where $I_{\text {initial }}$ is the source-drain current $I_{d s}$ measured after the scanning at $V_{t i p, p o l}=0 \mathrm{~V}$. This definition of the polarization-induced current rests on the fact that the P(VDF-TrFE) cannot be polarized at $0 \mathrm{~V}$ poling voltage; therefore, $I_{p o l}=0$. For both the reference and the nanoimprinted FeFET, $I_{p o l}$ is plotted in Figure 5 versus $V_{d s}$ (after scanning and removal of $V_{\text {tip,pol }}$ ), and in Figure 6 versus the previously applied $V_{t i p, p o l}$ at $V_{d s}=10 \mathrm{~V}$.

Figure 5 shows the output characteristics of a reference and a nanoimprinted FeFET. $I_{p o l}$ increases linearly with increasing $V_{d s}$ for source-drain voltages lower than $15 \mathrm{~V}$, for both FeFETs. The different curves coincide for poling voltages $V_{t i p, p o l}$ in absolute value larger than $30 \mathrm{~V}$ for the 

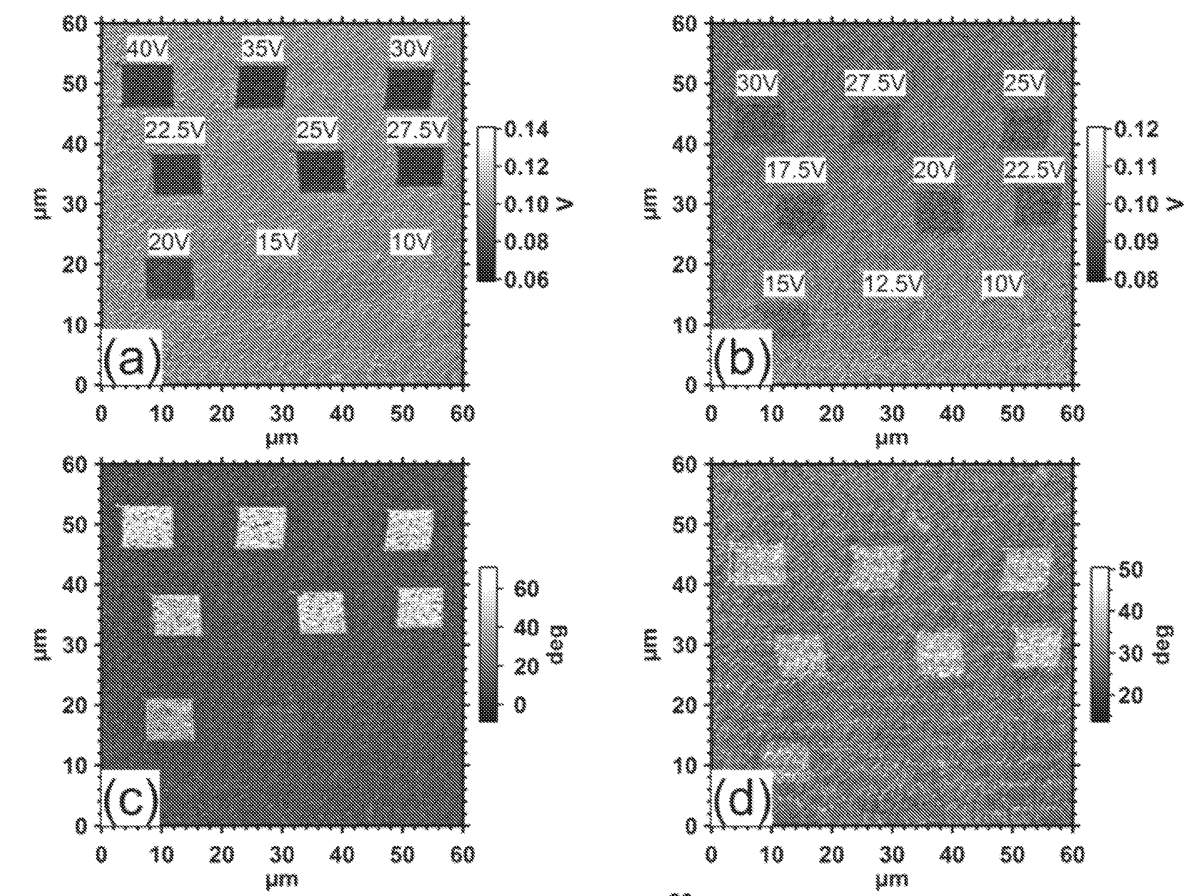

FIG. 4. PFM piezoresponse (a) and (b) amplitude images, (c) and (d) phase images: (a) and (c) for the continuous $\mathrm{P}(\mathrm{VDF}-\mathrm{TrFE})$ in a reference FcFET and (b) and (d) for the nanoimprinted $\mathrm{P}$ (VDF-TrFE) in a nanoimprinted FeFET. In all the images the background was polarized initially with a dc voltage of $0 \mathrm{~V}$, and the smaller square areas of $8 \mu \mathrm{m} \times 8 \mu \mathrm{m}$ were polarized with increasing positive dc voltages $V_{s \& d}$ applied on source-drain electrodes (given in panels (a) and (b)). All PFM images were recorded with a $0 \mathrm{~V}$ dc voltage; (e) and (f) PFM piezoresponse phase contrast versus source-drain voltage $V_{s \notin d}$ : (e) for the continuous P(VDF-TrFE) and (f) for
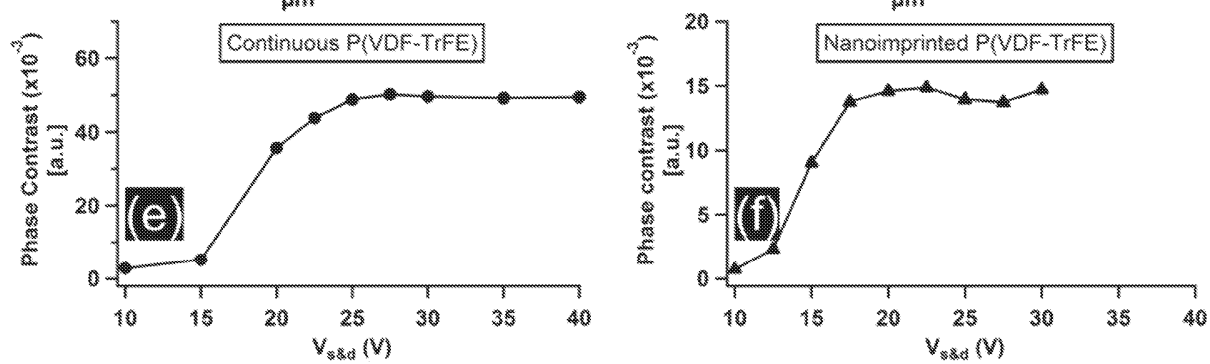
the nanoimprinted $\mathrm{P}(\mathrm{VDF}-\mathrm{TrFE})$.

reference FeFET and larger than $20 \mathrm{~V}$ for the nanoimprinted FeFET, confirming that the maximum $P_{r}$ is reached for $V_{\text {tip,pol }}=-30 \mathrm{~V}$ and $-20 \mathrm{~V}$ for the reference and nanoimprinted FeFET, respectively.

Figure 6 presents the transfer characteristics with a clear hysteresis for both the reference and the nanoimprinted FeFETs. $I_{\text {pol }}$ increases with decreasing $V_{\text {tip,pol }}$ starting from $0 \mathrm{~V}$, and saturates below $V_{\text {tip,pol }}=-30 \mathrm{~V}$ and $-20 \mathrm{~V}$ for the reference and nanoimprinted FeFET, respectively. This again confirms that the maximum polarization is reached faster for the nanoimprinted FeFET, which is consistent with the previous observation: the minimum absolute value of $V_{t i p, p o l}$ needed to obtain the saturation value of $I_{d s}$ is decreased by a factor of $c a .1 .5$ in the imprinted FeFET. This reduction is a significant improvement for the performance of the FeFET memory. When sweeping back $V_{\text {tip,pol }}$ (Figure 6), $I_{\text {pol }}$ keeps its saturation until $V_{\text {tip.pol }}$ reaches $15 \mathrm{~V}$ for the reference FeFET and $10 \mathrm{~V}$ for the nanoimprinted FeFET. At this stage, $l_{\text {pol }}$ starts to decrease, due to the switching of the direction of the P(VDF-TrFE) dipoles. Above $V_{\text {tip,pol }}=25 \mathrm{~V}$ and $20 \mathrm{~V}$ for the reference and nanoimprinted FeFETs, respectively, $I_{p o l}$ goes back close to its initial 0 value, corresponding to a random orientation state for the dipoles of P(VDF-TrFE). The lack of permanently aligned polarization after the application
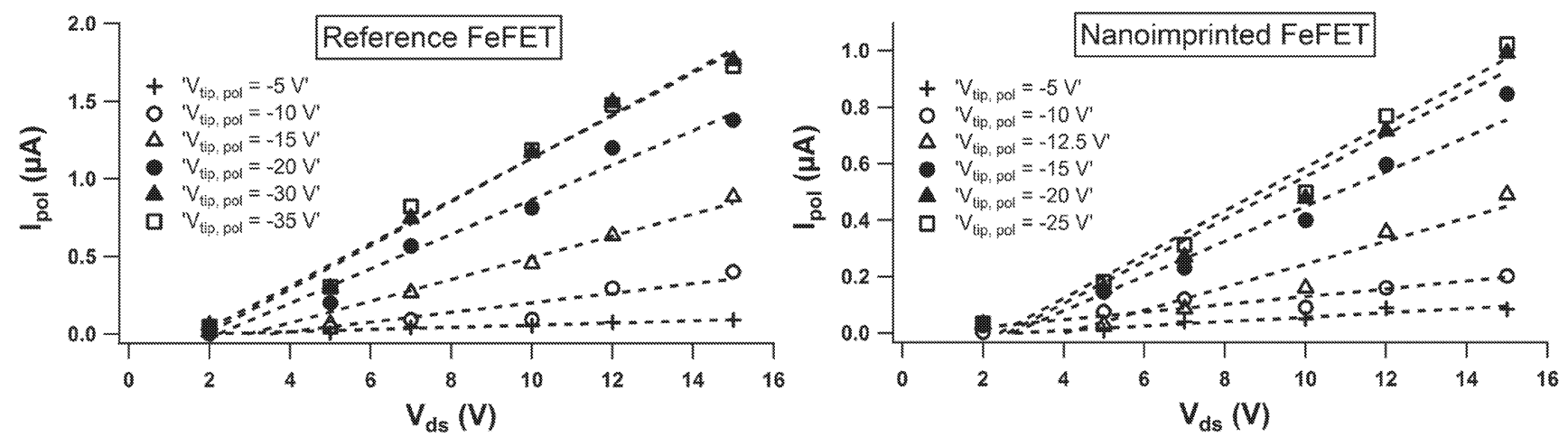

FIG. 5. Polarization-induced current $l_{p o l}$ (upon removal of $V_{t i p}$, pol) versus $V_{d s}$, for a reference and a nanoimprinted FeFET. The tip electrode scanning size for both FeFETs was $50 \mu \mathrm{m} \times 50 \mu \mathrm{m}$. Because the fill factor of the mold is 0.5 , the equivalent active area is $25 \mu \mathrm{m} \times 25 \mu \mathrm{m}$ for the nanoimprinted FeFET. 


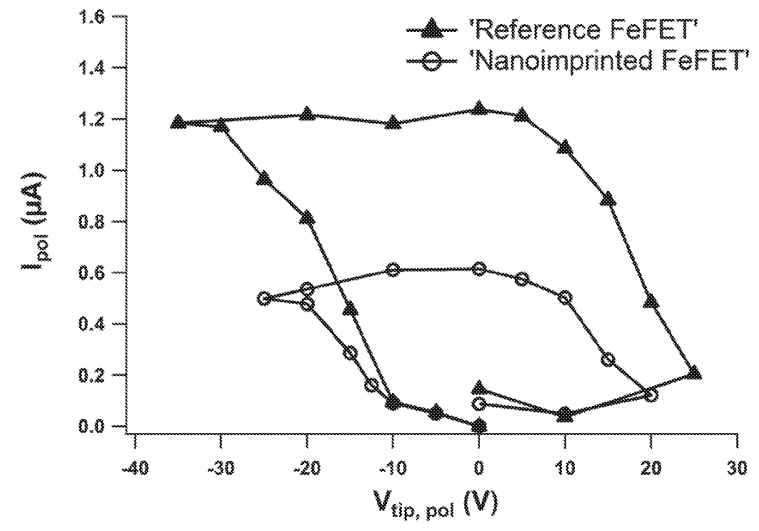

FIG. 6. Transfer characteristics of a reference and a nanoimprinted FeFET. The polarization-induced current $I_{p o l}$ was measured at $V_{d s}=10 \mathrm{~V}$ upon removal of $V_{t i p}$, pol. The tip electrode scaming size for both FeFETs was $50 \mu \mathrm{m} \times 50 \mu \mathrm{m}$. Because the fill factor of the mold is 0.5 , the equivalent active area is $25 \mu \mathrm{m} \times 25 \mu \mathrm{m}$ for the nanoimprinted FeFET. The measurement was started at $V_{\text {tip }, p o l}=0 \mathrm{~V}$, performed with a clockwise sequence, and finished again at $V_{t i p, p o l}=0 \mathrm{~V}$.

of a positive $V_{\text {tip,pol }}$ is due to the depolarization of the ferroelectrics in the absence of stabilizing negative charges in the p-type semiconductor layer, resulting in $I_{p o l}$ close to $0 .^{23}$

We noticed that the saturation value of $I_{p o l}$ and the ON/ OFF current ratio for the reference FeFET are $c a .2 .5$ times larger than those of the nanoimprinted FeFET. This is because the active area of the nanoimprinted FeFET is half of that of the reference FeFET, since the fill factor of the mold is 0.5 (the defects of nanoimprinted P(VDF-TrFE) shown in Figure $3(d)$ are probably responsible for a supplementary small reduction of current). Therefore, each P(VDF-TrFE) nanostripe acts more or less in isolation from its neighbors, showing that the transistor which we made actually consists of a series of nanostripe transistors placed in parallel. Hence, the system is potentially suited for a reduction of memory size, possibly down to one single nanostripe. This would however require a complex integration of the gate electrodes, which was not attempted here.

Further improvements might be obtained by decreasing the thickness of the ferroelectric nanostripes, or increasing the confinement of the polymer to decrease further the coercive field. ${ }^{18,19}$ In parallel, the fill factor of the mold could be increased for maximizing the current; alternatively, selecting a semiconductor of higher mobility would also improve the current. For final integration, the deposition of an insulating layer in the open grooves of the ferroelectric layer should be performed. These possible improvements will be investigated in further work.

In conclusion, we have integrated NIL in the fabrication process of a top-gate bottom-contact organic FeFET. A buried source-drain substrate was designed for the fabrication process. P(VDF-TrFE) imprinted in complete confinement on top of PTAA showed a decreased switching field compared to a continuous reference. In addition, the electric field needed to maximize the remnant polarization $P_{r}$ was significantly decreased by a factor of $c a .1 .5$ compared to a continuous film. A current characterization was performed by using a conductive PFM cantilever as gate electrode. Our results show that the nanoimprinted FeFET needs a smaller gate voltage to reach the maximum polarization-induced current, compared to the homogeneous reference. This leads to a decreased operating voltage for the memory application. Since our fabricated transistor consists of a large number of nanostripe-gated transistors placed in parallel, it also offers interesting possibilities for a strong size reduction of organic FeFETs.

Financial support was provided by the EU Program FP7/ 2007-2013 (MOMA, Agreement No. 248092), the Belgian Federal Science Policy (IAP P7/05), the F.R.S.-FNRS, and the Louvain Foundation (Mécénat Solvay).

${ }^{1}$ T. J. Reece, S. Ducharme, A. V. Sorokin, and M. Poulsen, Appl. Phys. Lett. 82(1), 142 (2003).

${ }^{2}$ K. N. Narayanan Unni, R. de Bettignies, S. Dabos-Seignon, and J.-M. Nunzi, Appl. Phys. Lett. 85(10), 1823 (2004).

${ }^{3}$ S. H. Lim, A. C. Rastogi, and S. B. Desu, J. Appl. Phys. 96(10), 5673 (2004).

${ }^{4}$ R. C. G. Naber, C. Tanase, P. W. M. Blom, G. H. Gelinck, A. W. Marsman, F. J. Touwslager, S. Setayesh, and D. M. de Leeuw, Nat. Mater. 4(3), 243 (2005).

${ }^{5}$ R. C. G. Naber, B. de Boer, P. W. M. Blom, and D. M. de Leeuw, Appl. Phys. Lett. 87(20), 203509 (2005).

${ }^{6}$ G. H. Gelinck, A. W. Marsman, F. J. Touwslager, S. Setayesh, D. M. de Leeuw, R. C. G. Naber, and P. W. M. Blom, Appl. Plyys. Lett. 87(9), 092903 (2005).

${ }^{7}$ R. C. G. Naber, M. Mulder, B. de Boer, P. W. M. Blom, and D. M. de Leeuw, Org. Electron. 7(3), 132 (2006).

${ }^{8}$ R. C. G. Naber, J. Massolt, M. Spijkman, K. Asadi, P. W. M. Blom, and D. M. de Leeuw, Appl Phys. Lett. 90(11), 113509 (2007).

${ }^{9}$ C. A. Nguyen, S. G. Mhaisalkar, J. Ma, and P. S. Lee, Org. Electron. 9(6), 1087 (2008).

${ }^{10}$ I. Lazareva, Y. Koval, P. Müller, K. Müller, K. Henkel, and D. Schmeisser, J. Appl. Phys. 105(5), 054110 (2009).

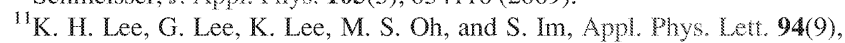
093304 (2009).

${ }^{12}$ S. Das and J. Appenzeller, Nano Let. 11(9), 4003 (2011).

${ }^{13}$ B. Kam, X. Li, C. Cristoferi, E. C. P. Smits, A. Mityashin, S. Schols, J. Genoe, G. Gelinck, and P. Heremans, Appl. Phys. Lett. 101(3), 033304 (2012).

${ }^{14}$ A. van Breemen, B. Kam, B. Cobb, F. G. Rodriguez, G. van Heck, K. Myny, A. Marrani, V. Vinciguerra, and G. Gelinck, Org. Electron. 14(8), $1966(2013)$.

${ }^{15}$ G. Knotts, A. Bhaumik, K. Ghosh, and S. Guha, Appl. Phys. Lett. 104(23), 233301 (2014).

${ }^{16}$ S. Ju. Kang, I. Bae, Y. J. Park, T. Ho. Park, J. Sung, S. C. Yoon, K. H. Kim, D. H. Choi, and C. Park, Adv. Funct. Mater. 19(10), 1609 (2009).

${ }^{17}$ H. J. Lee, Y.-J. Kim, E. Lee, K. Yao, and Ji. Y. Jo, Org. Electron. 15(3), 751 (2014).

${ }^{18}$ Z. Hu, M. Tian, B. Nysten, and A. M. Jonas, Nat. Mater. 8(1), 62 (2009).

${ }^{19}$ H. G. Kassa, R. Cai, A. Marrani, B. Nysten, Z. Hu, and A. M. Jonas, Macromolecules 46(21), 8569 (2013).

${ }^{20}$ S. Ju. Kang, I. Bae, Yu. J. Shin, Y. J. Park, J. Huh, S.-M. Park, Ho.-C. Kim, and C. Park, Nano Letr. 11(1), 138 (2011).

${ }^{21}$ Y. Wu, Q. Gu, G. Ding, F. Tong, Z. Hu, and A. M. Jonas, ACS Macro Lett. 2(6), 535 (2013).

${ }^{22}$ J. L. Lutkenhaus, K. McEnnis, A. Serghei, and T. P. Russell, Macromolecules 43(8), 3844 (2010).

${ }^{23}$ See supplementary material at htp $/ / \mathrm{dx}$.doi.org/10.1063/1.4896292 for detailed mold geometry, Figure S1 and for the demonstration and comment of the depolarization of the ferroelectrics after application of a positive poling voltage, Figure $\mathrm{S} 2$. 